\title{
ASYMPTOTIC EXPANSIONS OF THE WHITTAKER FUNCTIONS FOR LARGE ORDER PARAMETER
}

\author{
José L. López \\ Dedicated to Professor Richard Askey on the occasion of his 65th birthday.
}

\begin{abstract}
The asymptotic behavior of the Whittaker functions $M_{\kappa, \mu}(z)$ and $W_{\kappa, \mu}(z)$ for large modulus of the parameter $\kappa$ is considered. Asymptotic expansions in descending powers of $\sqrt{\kappa}$ are derived. The $\kappa$-independent coefficients of these expansions can be calculated in a simple way, making these approximations quite useful in practice. An explicit error bound for the expansion of $M_{\kappa, \mu}(z)$ also is obtained.
\end{abstract}

\section{Introduction}

The Whittaker functions $M_{\kappa, \mu}(z)$ and $W_{\kappa, \mu}(z)$ have acquired an ever increasing significance due to their frequent use in applications of mathematics to physical and technical problems. Most of their known properties are collected in monographs [4], [12], and in general treatises on special functions [2], [6 Vol. 2], [14].

Important research work has been developed during recent decades for obtaining asymptotic approximations of $M_{\kappa, \mu}(z)$ and $W_{\kappa, \mu}(z)$ for large values of the variable $z$ and/or the parameters $\kappa$ and $\mu$. A quite complete survey of the results obtained before 1975 can be found in [10] and references therein. It was pointed out in that article that there were several unsolved problems in this field at that time. One of these challenges was to obtain uniform asymptotic approximations for the case $|\mu| \rightarrow \infty$. This problem was satisfactory solved independently by Temme [13] and Olver [11]. They obtained uniform asymptotic approximations of these functions with respect to unrestricted values of $z \in(0, \infty)$ and bounded real values of $\kappa / \mu$ in terms of parabolic cylinder functions. A second outstanding problem was the case $|\kappa| \rightarrow \infty$. This task has been solved more recently by Dunster [5]. He obtained an asymptotic approximation of $M_{\kappa, \mu}(z)$ and $W_{\kappa, \mu}(z)$ for large real values of $\kappa$ in terms of Airy and Bessel functions. These expansions are uniformly valid for real values of $\kappa$ and $\mu$ verifying $0 \leq \mu / \kappa<1$.

The uniformity of the expansion is an important theoretical property, but, as a general rule, uniform asymptotic expansions are much more involved than nonuniform expansions. In this way, the uniform asymptotic expansions proposed by Temme [13] and Olver [11] for the Whittaker functions for large $|\mu|$ do not have an easy practical implementation. The coefficients of these expansions must be obtained by means of intricate equations that involve the asymptotic variable $\mu$. Something similar happens with the uniform asymptotic expansions proposed by Dunster [5] for large $|\kappa|$. The

Received March 28, 1998, revised February 15, 1999.

1991 Mathematics Subject Classification: 33C15, 41A60.

Key words and phrases: Whittaker functions, asymptotic expansions. 
coefficients of these expansions must be obtained by means of intricate equations that involve the asymptotic variable $\kappa$.

Therefore, for practical computations, simplicity of the expansion may be a more important property than uniformity. Much simpler asymptotic expansions (although not uniform) of the Whittaker functions for large $|\mu|$ and bounded values of $\kappa$ and $z$ can be found, for example, in $[4, \S 7.2]$. There, we can find cleaner expansions in inverse powers of $\mu$ whose $\mu$-independent coefficients are computed in an easy way. On the other hand, a clean expansion in inverse powers of $\kappa$ with $\kappa$-independent coefficients is not available in the literature.

In this paper we obtain asymptotic expansions of $M_{\kappa, \mu}(z)$ and $W_{\kappa, \mu}(z)$ in the sequence $\kappa^{-n / 2}$ for bounded values of $\mu$ and $z .^{1}$ The coefficients of the expansion are $\kappa$-independent and can be obtained in a systematic way. These properties make the expansions quite useful for practical calculations. The expansion of $M_{\kappa, \mu}(z)$ is accompanied also by an error bound.

As a disadvantage over the expansion given in [5], the expansion presented here is not uniform. As an analytic advantage, it is a clean expansion in inverse powers of $\sqrt{\kappa}$ with $\kappa$-independent and easily calculable coefficients. Besides, the expansion holds not only for real values of $\kappa$, but also for complex values.

In Section 2, the asymptotic expansion of $M_{\kappa, \mu}(z)$ in the sequence $\kappa^{-n / 2}$ and a bound for the remainder are derived. This expansion then is used to obtain an asymptotic approximation of $W_{\kappa, \mu}(z)$ for large $\kappa$. A brief summary is postponed to Section 3.

\section{Expansions of $M_{\kappa, \mu}(z)$ and $W_{\kappa, \mu}(z)$ in descending half-integer powers of $\kappa$}

The starting point is a convergent expansion of the Whittaker function $M_{\kappa, \mu}(z)$ in series of Bessel functions given by Buchholz [4, §7, Eq. (16)]. It reads

$$
M_{\kappa, \mu}(z)=\Gamma(2 \mu+1) 2^{2 \mu} z^{\mu+\frac{1}{2}} \sum_{n=0}^{\infty} p_{n}^{(2 \mu)}(z) \frac{J_{2 \mu+n}(2 \sqrt{z \kappa})}{(2 \sqrt{z \kappa})^{2 \mu+n}}
$$

where the $p_{n}^{(2 \mu)}(z)$ represent polynomials in $z^{2}$, which we call Buchholz polynomials. These are defined by

$$
p_{n}^{(\nu)}(z)=\frac{(i z)^{n}}{2 \pi i} \int^{(0+)} \exp \left(\frac{i z}{2}\left(\cot v-\frac{1}{v}\right)\right)\left(\frac{\sin v}{v}\right)^{\nu-1} \frac{d v}{v^{n+1}},
$$

and generated by the function

$$
\exp \left(\frac{-z}{2}\left(\operatorname{coth} t-\frac{1}{t}\right)\right)\left(\frac{\sinh t}{t}\right)^{\nu-1}=\sum_{n=0}^{\infty} p_{n}^{(\nu)}(z)\left(-\frac{t}{z}\right)^{n} .
$$

They also can be written in the form [1]

$$
p_{n}^{(\nu)}(z)=\frac{(i z)^{n}}{n !} \sum_{k=0}^{[n / 2]}\left(\begin{array}{c}
n \\
2 k
\end{array}\right) f_{k}^{(\nu)} g_{n-2 k}(z),
$$

\footnotetext{
${ }^{1}$ The asymptotic expansion of $M_{\kappa, \mu}(z)$ in inverse powers of $\sqrt{\kappa}$ has been obtained previously in [8], but only for certain ranges of values of $\kappa$ and $z$.
} 
as a sum of products of polynomials in $\nu$ and in $z$, separately, easily obtainable by means of the recurrence relations

$$
\begin{gathered}
f_{0}^{(\nu)}=1, \quad f_{m}^{(\nu)}=\frac{1-\nu}{2} \sum_{k=0}^{m-1}\left(\begin{array}{c}
2 m-1 \\
2 k
\end{array}\right) \frac{4^{m-k}\left|B_{2(m-k)}\right|}{m-k} f_{k}^{(\nu)}, \\
g_{0}(z)=1, \quad g_{m}(z)=-\frac{i z}{4} \sum_{k=0}^{[(m-1) / 2]}\left(\begin{array}{c}
m-1 \\
2 k
\end{array}\right) \frac{4^{k+1}\left|B_{2(k+1)}\right|}{k+1} g_{m-2 k-1}(z)
\end{gathered}
$$

where the $B_{2 n}$ denote the Bernoulli numbers [2, Table 23.2]. In what follows, $\mu \in$ $\mathbb{C} \backslash-\frac{1}{2} N$, the set $-\frac{1}{2} N=\left\{-\frac{1}{2},-1,-\frac{3}{2},-2, \ldots,\right\}$ being excluded since $M_{\kappa, \mu}(z)$ and $W_{\kappa, \mu}(z)$ are irregular at those values of $\mu$. Besides, we consider for $z, \mu$, and $\kappa$ only the main argument belonging to the sector $(-\pi, \pi]$. For obtaining the asymptotic expansion of $M_{\kappa, \mu}(z)$ in inverse powers of $\sqrt{\kappa}$, we will need the following lemmas.

Lemma 1. The Buchholz polynomials are bounded in the form

$$
\left|p_{n}^{(\nu}(z)\right| \leq \frac{|z|^{n}}{2^{n}} \sigma(\nu) e^{a|z|+2 \pi|\operatorname{Im}(\nu)|}
$$

where

$$
a=\frac{1}{4}\left[1+\frac{\cosh (2)}{2 \sin (2)}\right], \quad \sigma(\nu)= \begin{cases}\left(\frac{\cosh (2)}{2}\right)^{\operatorname{Re}(\nu)-1} & \text { if } \operatorname{Re}(\nu) \geq 1 \\ \left(\frac{\sin (2)}{2}\right)^{\operatorname{Re}(\nu)-1} & \text { if } \operatorname{Re}(\nu)<1\end{cases}
$$

Proof. If we choose the contour $|v|=2$ in the definition (2) of Buchholz polynomials, we obtain

where

$$
\left|p_{n}^{(\nu)}(z)\right| \leq I^{(\nu)}(z) \frac{|z|^{n}}{2^{n}}
$$

$$
I^{(\nu)}(z)=\frac{1}{4 \pi} \int_{|v|=2}\left|\exp \left\{\frac{i z}{2}\left(\cot v-\frac{1}{v}\right)\right\}\right|\left|\left(\frac{\sin v}{v}\right)^{\nu-1}\right||d v| .
$$

On the circle $|v|=2$, we have

$$
|\cos (v)| \leq \cosh (2), \quad \sin (2) \leq|\sin (v)| \leq \cosh (2) .
$$

Using these inequalities to bound the integrand in (10), equations (7) and (8) follow trivially.

Lemma 2. The Bessel functions satisfy the inequality

$$
\left|J_{2 \mu+n}(z)\right| \leq e^{5 \pi|\operatorname{Im}(\mu)|}\left(e^{\operatorname{Im}(z)}+e^{-\operatorname{Im}(z)}+\frac{\alpha_{n}(\mu, z)}{\pi}\right), \quad n=1,2,3, \ldots,
$$

where

$$
\alpha_{n}(\mu, z) \equiv\left\{\begin{array}{cl}
1 & \text { if } 2 \operatorname{Re}(\mu) \geq 1-n, \\
\epsilon^{-1} & \text { if } \operatorname{Re}(\mu) \geq-1,|z| \neq 0, \\
e^{\epsilon} \Gamma(-1-2 \operatorname{Re}(\mu)) \epsilon^{2 \operatorname{Re}(\mu)+1} & \text { if } \operatorname{Re}(\mu) \leq-1,|z| \neq 0,
\end{array}\right.
$$

and $\epsilon$ is any number satisfying $0<2 \epsilon \leq|\operatorname{Re}(z)|$ if $\operatorname{Re}(z) \neq 0$ or $0<2 \epsilon \leq|\operatorname{Im}(z)|$ if $\operatorname{Re}(z)=0$. 
Proof. From [2, Eqs. 9.1.22, 9.6.3, and 9.6.20], we have

$$
\begin{aligned}
J_{2 \mu+n}(z)=\frac{1}{\pi} \int_{0}^{\pi} \cos (z \sin \theta-(2 \mu+n) \theta) d \theta & \\
& -\frac{\sin (\pi(2 \mu+n))}{\pi} \int_{0}^{\infty} e^{-z \sinh (t)-(2 \mu+n) t} d t, \quad|\operatorname{Arg}(z)|<\frac{\pi}{2}, \\
J_{2 \mu+n}\left(x e^{i \pi / 2}\right)= & e^{i \pi(2 \mu+n) / 2\left[\frac{1}{\pi} \int_{0}^{\pi} e^{x \cos \theta} \cos ((2 \mu+n) \theta) d \theta\right.} \\
& \left.-\frac{\sin (\pi(2 \mu+n))}{\pi} \int_{0}^{\infty} e^{-x \cosh (t)-(2 \mu+n) t} d t\right], \quad x \geq 0 .
\end{aligned}
$$

On the one hand, for $2 \operatorname{Re}(\mu)+n \geq 1$, we can bound the integrand in the second integral of (14) by using $\sinh (t) \geq 0$ and $\left|e^{-(2 \mu+n) t}\right| \leq e^{-t}$. On the other hand, we use $e^{-n t} \leq e^{-t}$ in the second integral of (14), perform the change of variable $y=\operatorname{Re}(z)\left(e^{t}-1\right) / 2$, and bound the integrand by using $y / \operatorname{Re}(z) \geq 0$ if $1+\operatorname{Re}(\mu) \geq 0$ and $\operatorname{Re}(z) \geq 2 \epsilon$ if $1+\operatorname{Re}(\mu) \leq 0$. A bound for the integrand in the second integral of (15) is obtained in a similar way, but using $\cosh (t) \geq \sinh (t)$ and $x=\operatorname{Im}(z)$ instead of $\operatorname{Re}(z)$. Bounding also the integrands in the first integrals of (14) and (15), we obtain

$$
\begin{array}{r}
\left|J_{2 \mu+n}(z)\right| \leq e^{2 \pi|\operatorname{Im}(\mu)|}\left(e^{\operatorname{Im}(z)}+e^{-\operatorname{Im}(z)}+\frac{\alpha_{n}(\mu, z)}{\pi}\right), \quad \operatorname{Re}(z) \geq \epsilon>0, \\
\left|J_{2 \mu+n}\left(x e^{i \pi / 2}\right)\right| \leq e^{3 \pi|\operatorname{Im}(\mu)|}\left(e^{x}+e^{-x}+\frac{\alpha_{n}(\mu, x)}{\pi}\right), \quad x \geq \epsilon>0 .
\end{array}
$$

Finally, using the analytic continuation property [2, Eq. 9.1.35] of the Bessel functions,
we obtain (12) and (13).

Theorem 2.1. For bounded values of $z \neq 0$ and $\mu$, large values of $|\kappa|$, and the sector $|\operatorname{Arg}(z)+\operatorname{Arg}(\kappa)|<2 \pi$, the Whittaker function $M_{\kappa, \mu}(z)$ admits the asymptotic
expansion

$$
\begin{aligned}
M_{\kappa, \mu}(z)= & \frac{\Gamma(2 \mu+1) z^{\frac{1}{4}}}{\sqrt{\pi} \kappa^{\mu+\frac{1}{4}}}\left[\cos \left(2 \sqrt{z \kappa}-\mu \pi-\frac{\pi}{4}\right) \sum_{n=0}^{\lfloor N / 2\rfloor} \frac{M_{2 n}(\mu, z)}{(-\kappa)^{n}}\right. \\
& \left.-\frac{1}{\sqrt{\kappa}} \sin \left(2 \sqrt{z \kappa}-\mu \pi-\frac{\pi}{4}\right) \sum_{n=0}^{\lfloor(N-1) / 2\rfloor} \frac{M_{2 n+1}(\mu, z)}{(-\kappa)^{n}}+R_{N}^{(\mu)}(z, \kappa)\right]
\end{aligned}
$$

where the coefficients of the expansion $M_{n}(\mu, z)$ are given by

$$
M_{n}(\mu, z)=\frac{1}{(4 \sqrt{z})^{n}} \sum_{m=0}^{n}(-2)^{m} p_{m}^{(2 \mu)}(z) \frac{(2 \mu-n+2 m+1 / 2)_{2(n-m)}}{(n-m) !} .
$$


For $|\kappa| \geq|z| / 4$ and the sector $|\operatorname{Arg}(z)+\operatorname{Arg}(\kappa)| \leq \pi$, the remainder term $R_{N}^{(\mu)}(z, \kappa)$ is an $\mathcal{O}\left(\kappa^{-N / 2-1 / 2}\right)$ quantity bounded by

$$
\begin{aligned}
\left|R_{N}^{(\mu)}(z, \kappa)\right| \leq & {\left[\frac{\sqrt{\pi}}{2^{N}} \sum_{n=0}^{N} \frac{2^{n}\left|(2 \mu+2 n-N-1 / 2)_{2(N-n+1)}\right|}{(N-n+1) !} e^{|\operatorname{Im}(2 \sqrt{z \kappa}-\mu \pi)|}\right.} \\
& \times \frac{\Gamma((N-n) / 2+3 / 2)\left|p_{n}^{(2 \mu)}(z)\right|}{\Gamma((N-n) / 2+1)} \exp \left\{\frac{\pi\left|(2 \mu+n)^{2}-1 / 4\right|}{2|2 \sqrt{z \kappa}|}\right\} \\
+ & \left.\frac{2}{\sqrt{\pi}}|z \kappa|^{1 / 4} \sigma(2 \mu) e^{a|z|+10 \pi|\operatorname{Im} \mu|}\left(\pi+\alpha_{N+1}(\mu, 2 \sqrt{z \kappa})\right)\right]\left|\frac{\sqrt{z}}{2 \sqrt{\kappa}}\right|^{N+1}
\end{aligned}
$$

where $\alpha_{n}(\mu, z)$ is given by (13) and $\sigma(2 \mu)$ and a are given by (8).

Proof. From (1) and using $J_{\nu}(z)=\left(H_{\nu}^{(1)}(z)+H_{\nu}^{(2)}(z)\right) / 2$ where $H_{\nu}^{(1)}$ and $H_{\nu}^{(2)}$ represent the Hankel functions, we have

$$
\begin{aligned}
M_{\kappa, \mu}(z)=\Gamma(2 \mu+1) z^{\frac{1}{2}} \kappa^{-\mu} & {\left[\sum_{n=0}^{N} \frac{p_{n}^{(2 \mu)}(z)}{2(2 \sqrt{z \kappa})^{n}}\right.} \\
& \left.\times\left(H_{2 \mu+n}^{(1)}(2 \sqrt{z \kappa})+H_{2 \mu+n}^{(2)}(2 \sqrt{z \kappa})\right)+r_{N}^{(\mu)}(z, \kappa)\right]
\end{aligned}
$$

where

$$
r_{N}^{(\mu)}(z, \kappa)=\sum_{n=N+1}^{\infty} \frac{p_{n}^{(2 \mu)}(z)}{(2 \sqrt{z \kappa})^{n}} J_{2 \mu+n}(2 \sqrt{z \kappa}) .
$$

Now we can use the asymptotic expansions [9, Chap. 7, Eqs. (4.03) and (4.04)],

$$
\begin{aligned}
& H_{\nu}^{(1)}(t)=\sqrt{\frac{2}{\pi t}} e^{i\left(t-\nu \frac{\pi}{2}-\frac{\pi}{4}\right)}\left(\sum_{m=0}^{N} \frac{(-1)^{m}}{(2 i t)^{m}} \frac{\left(\nu-m+\frac{1}{2}\right)_{2 m}}{m !}+\eta_{N}^{(1)}(\nu, t)\right) \\
& H_{\nu}^{(2)}(t)=\sqrt{\frac{2}{\pi t}} e^{-i\left(t-\nu \frac{\pi}{2}-\frac{\pi}{4}\right)}\left(\sum_{m=0}^{N} \frac{1}{(2 i t)^{m}} \frac{\left(\nu-m+\frac{1}{2}\right)_{2 m}}{m !}+\eta_{N}^{(2)}(\nu, t)\right),
\end{aligned}
$$

simultaneously valid for all $\nu$ when $t$ is confined to the sector $|\operatorname{Arg}(t)|<\pi$. For $|\operatorname{Arg}(t)| \leq \pi / 2$, the error term $\eta_{N}^{(1)}(\nu, t)$ is an $\mathcal{O}\left(t^{-N-1}\right)$ quantity bounded by [9, Chap. 7, Eqs. (13.02)-(13.05)],

$$
\left|\eta_{N}^{(1)}(\nu, t)\right| \leq \frac{\sqrt{\pi}(\nu-N-1 / 2)_{2(N+1)} \Gamma(N / 2+3 / 2)}{2^{N}(N+1) ! \Gamma(N / 2+1)|t|^{N+1}} \exp \left\{\frac{\pi\left|\nu^{2}-1 / 4\right|}{2|t|}\right\}
$$

and the same holds for $\eta_{N}^{(2)}(\nu, t)$. Then, introducing (23) and (24) into equation (21) and grouping powers of $\kappa^{-1 / 2}$, we obtain, for $|\operatorname{Arg}(z)+\operatorname{Arg}(\kappa)|<2 \pi$,

$$
\begin{aligned}
& M_{\kappa, \mu}(z)=\frac{\Gamma(2 \mu+1) z^{\frac{1}{4}}}{2 \sqrt{\pi} \kappa^{\mu+\frac{1}{4}}}\left[e^{i\left(2 \sqrt{z \kappa}-\mu \pi-\frac{\pi}{4}\right)}\left(\sum_{n=0}^{N} \frac{(-1)^{n} M_{n}(\mu, z)}{(i \sqrt{\kappa})^{n}}+\rho_{N}^{(1)}(\mu, z, \kappa)\right)\right. \\
& \left.+e^{-i\left(2 \sqrt{z \kappa}-\mu \pi-\frac{\pi}{4}\right)}\left(\sum_{n=0}^{N} \frac{M_{n}(\mu, z)}{(i \sqrt{\kappa})^{n}}+\rho_{N}^{(2)}(\mu, z, \kappa)\right)+2 \pi^{1 / 2}(z \kappa)^{1 / 4} r_{N}^{(\mu)}(z, \kappa)\right]
\end{aligned}
$$


where the coefficients $M_{n}(\mu, z)$ are given in (19) and the remainder term $\rho_{N}^{(1)}(\mu, z, \kappa)$ is an $\mathcal{O}\left(\kappa^{-N / 2-1 / 2}\right)$ quantity bounded, for $|\operatorname{Arg}(z)+\operatorname{Arg}(\kappa)| \leq \pi$, by

$$
\begin{aligned}
\left|\rho_{N}^{(1)}(\mu, z, \kappa)\right| \leq \frac{\sqrt{\pi}}{2^{N}} \sum_{n=0}^{N} \frac{2^{n}\left|(2 \mu+2 n-N-1 / 2)_{2(N-n+1)}\right|}{\frac{\Gamma((N-n) / 2+3 / 2)}{\Gamma((N-n) / 2+1)}} & (N-n+1) ! \\
& \times\left|p_{n}^{(2 \mu)}(z)\right| \exp \left\{\frac{\pi\left|(2 \mu+n)^{2}-1 / 4\right|}{2|2 \sqrt{z \kappa}|}\right\} \frac{1}{|2 \sqrt{z \kappa}|^{N+1}}
\end{aligned}
$$

and the same holds for $\rho_{N}^{(2)}(\mu, z, \kappa)$. To prove $(18)-(20)$, we still have to show that $r_{N}^{(\mu)}(z, \kappa)$ in equation $(26)$ is bounded by an $\mathcal{O}\left(\kappa^{-(N / 2+1 / 2)}\right)$ quantity. But using Lemmas 1 and 2 , we have, for $|w| \geq|z|$ and $n \geq N+1$,

$$
\begin{aligned}
\left|\frac{p_{n}^{(2 \mu)}(z)}{w^{n}} J_{2 \mu+n}(w)\right| \leq \frac{1}{2^{n}} \sigma(2 \mu) & e^{a|z|+9 \pi|\operatorname{Im}(\mu)|} \\
& \times\left(e^{\operatorname{Im}(w)}+e^{-\operatorname{Im}(w)}+\frac{\alpha_{N+1}(\mu, w)}{\pi}\right)\left|\frac{z}{w}\right|^{N+1}
\end{aligned}
$$

where $\sigma(2 \mu)$ and $a$ are given by (8). Therefore, using the definition (22) of $r_{N}^{(\mu)}(z, \kappa)$ and this last inequality, we find that the remainder $r_{N}^{(\mu)}(z, \kappa)$ in the expansion (26) is bounded by

$$
\begin{aligned}
\left|r_{N}^{(\mu)}(z, \kappa)\right| \leq \sigma(2 \mu) & e^{a|z|+9 \pi \mid \operatorname{Im}(\mu)} \\
& \times\left(e^{\operatorname{Im}(2 \sqrt{z \kappa})}+e^{-\operatorname{Im}(2 \sqrt{z \kappa})}+\frac{\alpha_{N+1}(\mu, 2 \sqrt{z \kappa})}{\pi}\right)\left|\frac{\sqrt{z}}{2 \sqrt{\kappa}}\right|^{N+1} .
\end{aligned}
$$

After straightforward operations and using (27) and (29) in (26), we obtain (18) and (20).

A recursion relation for the coefficients $M_{n}(\mu, z)$ in the expansion (18) can be obtained from Olver's method of deriving asymptotic expansions from differential equations with a large parameter [9, Chap. 10]. The Whittaker differential equation can be written in the form

$$
\frac{d^{2} w}{d z^{2}}+\frac{\kappa}{z} w=g(z) w
$$

where $g(z)=\left(z^{2}+4 \mu^{2}-1\right)(2 z)^{-2}$. After the transformations $z=\zeta^{2} / 4$ and $W=$ $z^{-1 / 4} w$, we obtain

$$
\frac{d^{2} W}{d \zeta^{2}}+\kappa W=\psi(\zeta) W, \quad \psi(\zeta)=\frac{\zeta^{2}}{16}+\frac{4 \mu^{2}-1 / 4}{\zeta^{2}}
$$

The asymptotic expansion in the sequence $(\sqrt{\kappa})^{-n}$ of two independent solutions $W^{+}(\kappa, \zeta)$ and $W^{-}(\kappa, \zeta)$ (analytic for $z \neq 0$ ) of the above differential equation are given by [9, Chap. 10, Theorem 3.1]

$$
W^{ \pm}(\kappa, \zeta) \sim e^{ \pm i \sqrt{\kappa} \zeta} \sum_{k=0}^{\infty} \frac{M_{n}(\mu, z)}{(\mp i \sqrt{\kappa})^{n}}
$$


where the coefficients $M_{n}\left(\mu, \zeta^{2} / 4\right)$ are defined recursively by

$$
M_{n+1}\left(\mu, \zeta^{2} / 4\right)=\frac{1}{2} \frac{d M_{n}\left(\mu, \zeta^{2} / 4\right)}{d \zeta}-\frac{1}{2} \int \psi(\zeta) M_{n}\left(\mu, \zeta^{2} / 4\right) d \zeta,
$$

with $M_{0}\left(\mu, \zeta^{2} / 4\right)=1$. From the asymptotic behavior of $M_{\kappa, \mu}(z)$ for large $\kappa$, we see that

$$
M_{\kappa, \mu}(z)=\frac{\Gamma(2 \mu+1) z^{1 / 4}}{2 \sqrt{\pi} \kappa^{\mu+1 / 4}}\left(e^{-i(\mu \pi+\pi / 4)} W^{+}(\kappa, \zeta)+e^{i(\mu \pi+\pi / 4)} W^{-}(\kappa, \zeta)\right) .
$$

The coefficients of the expansion $M_{n}(\mu, z)$ given explicitly in (19) also are given by the recurrence (33) with the integration constants set to zero. Therefore, we obtain the expansion (18).

Olver's method also provides an error bound for the remainder terms in the expansions (32) and, therefore, for the remainder in the expansion of $M_{\kappa, \mu}(z)$. But, as a consequence of the divergence of the coefficients $M_{n}\left(\mu, \zeta^{2} / 4\right)$ for large $\zeta$, these bounds cannot be uniformly defined for arbitrarily large $z$ (see [9, Chap. 10, Theorem 3.1] for details).

An asymptotic approximation of $W_{\kappa, \mu}(z)$ can be obtained from the expansion (18) of $M_{\kappa, \mu}(z)$ and the relations [2, Eqs. (6.1.17) and (13.1.34)],

$$
\begin{aligned}
W_{\kappa, \mu}(z)=\frac{\Gamma(-2 \mu) \Gamma(\kappa+\mu+1 / 2)}{\pi} \cos [\pi(\kappa+\mu)] M_{\kappa, \mu}(z) & \\
+ & \frac{\Gamma(2 \mu) \Gamma(\kappa-\mu+1 / 2)}{\pi} \cos [\pi(\kappa-\mu)] M_{\kappa,-\mu}(z) .
\end{aligned}
$$

The expansion (18) has been obtained for $|\operatorname{Arg}(z)+\operatorname{Arg}(\kappa)|<2 \pi$ and the bound (20) for $|\operatorname{Arg}(z)+\operatorname{Arg}(\kappa)| \leq \pi$. We can extend these parameters to the whole complex plane by using the reflection formula [5, Eq. (2.11)],

$$
M_{-\kappa, \mu}\left(z e^{ \pm \pi i}\right)=e^{ \pm(\mu+1 / 2) \pi i} M_{\kappa, \mu}(z)
$$

\section{Summary}

For bounded values of $z \neq 0$ and $\mu$ and large values of $|\kappa|$, the asymptotic expansion of the Whittaker function $M_{\kappa, \mu}(z)$ in inverse powers of $\sqrt{\kappa}$ is given in equation (18) for $|\operatorname{Arg}(z)+\operatorname{Arg}(\kappa)|<2 \pi$. The coefficients of this expansion are given in equation (19) and satisfy the recurrence relation (33). A bound for the remainder after $N$ terms is given in equation (20) for $|\operatorname{Arg}(z)+\operatorname{Arg}(\kappa)| \leq \pi$. In order to cover the whole complex $z, \kappa$, and $\mu$-plane, equations (18) and (20) should be used combined with (36).

For bounded values of $z \neq 0$ and $\mu$ and large values of $|\kappa|$, an asymptotic approximation of the Whittaker function $W_{\kappa, \mu}(z)$ in inverse powers of $\sqrt{\kappa}$ can be obtained combining (18) and (35). The Stirling asymptotic expansions of the gamma functions in (35) should be introduced for obtaining an asymptotic approximation of $W_{\kappa, \mu}(z)$ in inverse powers of $\sqrt{\kappa}$ and $\kappa \pm \mu+1 / 2$.

Acknowledgments. The author is grateful to J. Sesma for enlightening discussions and to the anonymous referee for his/her suggestions. The financial support of Comisión Interministerial de Ciencia y Tecnología is acknowledged. 


\section{References}

1. J. Abad and J. Sesma, Computation of the regular confluent hypergeometric function, The Mathematica Journal 5 (1995), 74-76

2. M. Abramowitz and I. A. Stegun (Eds.), Handbook of Mathematical Functions, Dover, New York, 1965.

3. N. Bleistein and R. A. Handelsman, Asymptotic Expansions of Integrals, Dover Pub., New York, 1974 .

4. H. Buchholz, The Confluent Hypergeometric Function, Springer-Verlag, Berlin, 1969.

5. T. M. Dunster, Uniform asymptotic expansions for Whittaker's confluent hypergeometric functions, SIAM. J. Math. Anal. 29 (1989), 744-760.

6. A. Erdélyi (Ed.), Higher Transcendental Functions, McGraw-Hill, New York, 1953.

7. A. Erdélyi, Asymptotic Expansions, Dover Pub., New York, 1956.

8. J. L. López and J. Sesma, The Whittaker function $M_{\kappa, \mu}(z)$ as a function of $\kappa$, Constr. Approx. 15 (1999), 83-95.

9. F. W. J. Olver, Asymptotics and Special Functions, Academic Press, New York, 1974.

10. Unsolved problems in the asymptotic estimation of special functions. In: Theory and Applications of Special Functions, (R. Askey, ed.), Academic Press, New York, 1975, pp. 99-141.

11. Whittaker functions with both parameters large: uniform approximations in terms of parabolic cylinder functions, Proc. Roy. Soc. Edinburgh 86A (1980), 213-234.

12. L. J. Slater, Confluent Hypergeometric Functions, Cambridge Univ. Press, Cambridge, 1960.

13. N. M. Temme, Uniform asymptotic expansions of confluent hypergeometric functions, J. Inst. Math. Appl. 22 (1978), 215-223.

14. Special Functions: An Introduction to the Classical Functions of Mathematical Physics, John Wiley and Sons, New York, 1996.

Departamento de Matemática Aplicada, Facultad de Ciencias, Universidad de Zaragoza, 50009-Zaragoza, SPAIN

E-mail: jllopez@posta.unizar.es 